\title{
RESTAURACIÓN DE AMBIENTES DETERIORADOS DERIVADOS DE LA SELVA TROPICAL HÚMEDA: EL USO DE LOS HONGOS MICORRIZÓGENOS ARBUSCULARES
}

\author{
Javier Álvarez-Sánchez', Patricia Guadarrama, Irene Sánchez-Gallen y Diego Olivera \\ Departamento de Ecología y Recursos Naturales, Facultad de Ciencias, Universidad Nacional Autónoma de México, \\ Circuito Exterior s/n, Ciudad Universitaria, México 04510, D.F., México. \\ 'Autor para la correspondencia: Tel. (55) 5622-4835; correo-e: fjas@fciencias.unam.mx
}

\begin{abstract}
Resumen: Se analizó el efecto de hongos micorrizógenos arbusculares (HMA) sobre el crecimiento y la supervivencia de plántulas de una especie dependiente de luz (Piper auritum) y una tolerante a la sombra (Rollinia jimenezii), las cuales fueron trasplantadas a áreas deterioradas derivadas de una selva tropical húmeda en la región de Los Tuxtlas, Veracruz, México. En invemadero se realizó un ex pe rimento considerando tres factores: especie, micorrización (con o sin HMA) y suelo (de selva o de pastizal). Se hizo un análisis clásico de crecimiento para siete vari ables y los análisis de varianza correspondientes mostraron que el factor micomización produjo dife rencias signific ativas para el peso seco de hojas, la Proporción de Área Foliar (PAF) y la Tasa de Asimilación Neta (TAN); en especial la interacción especie $\times$ HMA fue una fuente muy importante de va riación. Después de 120 días, las plántulas fueron transplantadas a dos sitios en un pastizal donde se sembra ron al azar de acuerdo con el tratamiento; siete meses después fueron cosechadas. En este segundo experimento, Piper obtuvo las mayo res medias y fue dife rente significativamente deRollinia en casi todas las vari ables decrecimiento, mientras que el factor suelo no generó ninguna dife rencia significativa y el factor micorrización sólo produjo dife rencias en tres variables; el nivel sin HMA (M-) produjo los valores mayo res en la Tasa Relativa de Crecimiento (TRC) y Área Foliar Específica (AFE), y el nivel con HMA (M+) tuvo el mismo efecto en la TAN. La combinación especie $\times$ micorrización fue signific at iva y la combinación Piper sin micorrizas obtuvo la media con los valores mayores. La triple interacción fue solamente significativa para la TRC, siendo la media menor y diferente para Rollinia con micorrizas en suelo de selva. Sin embargo, la presencia de HMA pro movió la supervivencia en campo en las dos especies. El uso de especies pertenecientes a diferentes gremios permite tener mayo res posibilidades de éxito en la restauración, ya que tienen respuestas ecofisiológcas diferentes de acuerdo con las condiciones ambientales prevalecientes; el uso de HMA antes del transplante aseguró un mayor crecimiento en Piper pe rono en Rollinia, p e rola supervivencia en campo fue mayor para ambas con $\mathrm{M}+$; asimismo, en la mayoría de los casos el suelo de selva determinó la mayor respuesta en Rollinia, y el de pastizal en Piper.
\end{abstract} Palabras clave: dependiente de luz, hongos micorrizógenos arbusculares, restauración, selva húmeda, tolerante a la sombra.

\begin{abstract}
The influence of arbuscular my c o nhizal fungi (AMF) on the growth and surv ival of seedlings of a light dependent (Piper a uritum) and a shade tolerant (Rollinia jimenezii) tree species was analy zed in the context of degraded env i ronments derived from the tropical rain forest at Los Tuxtlas, Veracnuz, Mexico. An experiment with three factors, soil (from tropical rain fo rest or pasture), my corrhization (with or without AMF), and species was carried out in a greenhouse. A classical growth analysis was performed with seven variables, and ANOVAs showed significant differences for dry leaf weight, Leaf Area Ratio and Net Assimilation Rate (NAR); the AMF $\times$ species interaction was particularly important. After 120 days, seedlings we re transplanted into two sites within a pasture and randomly placed according to the tre atment factors; seedlings we re harvested after seven months. In this second experiment, Piper obtained the largest mean values and was significantly different from Rollinia, whe reas the soil factor did not produce any significant differences, and the factor mycorrhization only produced diffe rences in three variables; seedlings without AMF (M-) had the highest Relative Growth Rate (RGR) and Specific Leaf A rea (SLA) values, whereas those with the AMF $(\mathrm{M}+)$ tre atment had the largest TAN values. The AMF $\times$ species interaction was significant, the higher values being those obtained for Piper without AMF. The triple interaction was significant only for RGR, the mean being smaller and different forRollinia with my c o rhizae in fo rest soil. However, survival did increase under AMF tre atments for Rollinia and for Piper growing on pasture soil. We recommend the use of both pioneer and late successional species for ecological re sto ration; in particular, Piper and Rollinia seedlings should be inoculated with AMF, as this procedure increases their surv ival after transplanting.

Key words: Arbuscular mycorrhizal fungi, light dependent species, restoration, shade tolerant species, tropical rain forest.
\end{abstract}

a deforestación es la principal causa de la pérdida de la L vegetación en los ecosistemas tropicales. Este proceso se origina principalmente por la explotación forestal, la apertura de campos de cultivo y potreros, y el crecimiento y desarrollo de infraestructura urbana (Geist y Lambin, 2001, 2002). A nivel mundial, se ha estimado que los bosques tropicales están siendo destruidos a una tasa anual de 4\% de su extensión (Dobson et al., 1997). En México, 
durante la década de los ochenta, $75 \%$ de la deforestación ocumió en las selvas húmedas (Hughes et al., 2000; Guevara et al., 2004).

Una consecuencia inmediata de la defo restación es la disminución de la cobertura vegetal y la fragmentación de la vegetación original en parches de diferentes tamaños, que traen como resultado el deterioro biológico de dicha vegetación y el de las comunidades existentes en ella (Dirzo, 2001). Este deteri o rotiene dife rentes grados y se puede presentar en va rios niveles, observándose desde extinciones poblacionales locales hasta cambios en el ciclo del $\mathrm{CO}_{2}$ atmosférico, del agua y de los nut rientes (Lüttge, 1997).

La recuperación de un ambiente degradado como los potreros o las comunidades vegetales de los parches está determinada por varios factores. Uno de los más importantes, dado su papel fundamental en el desarrollo y permanencia de las comunidades vegetales (Wardle, 2002), es la biota edáfica ori ginal. En este sentido, un grupo muy relevante de organismos que habitan en el suelo y que se relacionan con las plantas son los hongos micorri zó genos arbu sculares (HMA). Estos hongos fo rman una asociación mu t ualista con las plantas, denominada micorriza arbuscular. $\mathrm{Su}$ función más importante es incrementar la absorción de nutrientes, sobre todo P y N (Smith y Read, 1997), y agua del suelo, lo que se re fleja en una mayor producción de biomasa y un mejor estado hídrico de las plantas, confiniéndoles ventajas ante condiciones estresantes como la falta de agua disponible o la presencia de pató genos y competidores (Varma y Hock, 1999); todo esto repercute en un mayor crecimiento, supervivencia y establecimiento vegetal. Una característica particular de los HMA es su potencial de inocular a una gran variedad de especies vegetales; sin embargo, en fe chas recientes se ha destacado la importancia de la identidad de las especies, tanto vegetales como fúngicas, ya que se ha encontrado que la magnitud de los beneficios que obtenga una especie de planta depende de la especie de hongo que la esté colonizando y viceversa, es decir, existe una respuesta dife rencial entre ambos simbiontes que determina en mu cho el éxito ecológico de ambas especies (Van der Heijden et al., 1998a, 1998b; Van der Hart et al., 2003).

La recuperación de sitios de selva húmeda degradados utilizando HMA es una vía poco explorada que resulta muy atractiva por todas las ventajas mencionadas que conllevan dichos hongos. La inoculación de HMA provenientes de la vegetación ori ginal previa al transplante en campo puede subsanar la falta de éstos en los ambientes deteriorados, ya que algunos estudios sobre el efecto de la deforestación y la fragmentación sobre la comunidad de los HMA han repo rtado que en algunas zonas de pastizal tropical su dive rsidad disminu ye, mientras que su abu ndancia de esporas aumenta, en comparación con la selva colindante (Cuenca et al., 1998a, 1998b); en contraste, ot ros estudios no muestran cambios en la dive rsidad y riqueza entre pastizales y selva (Picone, 2000) o entre fragmentos grandes y pequeños (Mangan et al., 2004), perosí en su composición.

Una alternativa para solucionar la degradación ambiental en las selvas húmedas sería no inducir ningún cambio, permitiendo que los sitios degradados y abandonados se regeneren naturalmente, pero este proceso es muy lento. Otra posibilidad es la intervención que consiste en que, una vez identificados algunos procesos y/o componentes específicos críticos para la recuperación de tales sitios en el contexto de la sucesión ecológica, puedan ser manipulados de manera artificial, acelerándolos y alcanzando la restauración ecológica de la selva (Dobson et al., 1997).

La restauración ecológica se define como "el proceso de asistencia para la recuperación de un ecosistema que ha sido degradado, dañado o destruido" (SER, 2004). Ésta debe tomar en cuenta un intervalo crítico de variabilidad de la biodiversidad y de los procesos ecológicos, haciendo énfasis en la sucesión ecológica y en los at ri butos de historia de vida de las especies (Cuenca et al., 2004; Hérault et al., 2005), y tratando de rep roducir un ecosistema de referencia (Aronson y Le Floc'h, 1996; Hobbs y Norton, 1996; Young, 2000; Maluf de Souza y Ferreira, 2004).

Para alcanzar estos objetivos, es recomendable, por un lado, elabora un programa para el establecimiento de una comunidad vegetal que pueda madurar en el sitio, que contemple la reintroducción de especies clave de los diferentes estadios sucesionales, junto con especies que hayan permanecido después del disturbio (Bradshaw, 1997) o que arriben después de él. Por otro lado, es deseable llevar a cab o una recuperación del suelo debido a que el establecimiento de un sistema funcional estable a largo plazo requiere del desamollo de una microbiota nativa (Perry y Amaranthus, 1990; Haselwandter, 1997), todo con el fin de constituir un ecosistema lo más parecido al que existió antes de los disturbios.

En los sistemas tropicales húmedos, la mayoría de las especies vegetales estudiadas están asociadas con los HMA (Varela y Guadarrama, 2003), los cuales influyen en el restablecimiento de las especies vegetales al incrementar su desempeño (Janos, 1996). Por ello, la restauración de estos ecosistemas utilizando HMA tendrá como resultado un exitoso establecimiento y un mejor crecimiento vegetal (Siqueira et al., 1998), y facilitará la determinación de la dirección del proceso sucesional (Aziz et al., 1995).

El objetivo de este estudio fue analizar la supervivencia y el crecimiento de dos especies (una dependiente de luz y otra tolerante a la sombra) de una selva húmeda, utilizando los hongos micorrizógenos arbusculares como una herramienta para que, a partir de ello, se establezcan las pri me ras pautas para la restauración ecológica de esta selva.

\section{Área de estudio}

Este estudio se realizó en un invernadero ubicado en la Estación de Biología Tropical "Los Tuxtlas" del Instituto 
de Biología (Universidad Nacional Autónoma de México) y en un pastizal cercano que ocupó su lugar luego del cambio por un uso de suelo a potrero, donde existen algunos árboles remanentes.

La Estación "Los Tuxtlas" se localiza en el estado de Veracruz, a altitudes de 150 a 530 m s.n.m. (GónzalezSoriano et al., 1997). El clima es cálido-húmedo (García, 1988), la precipitación total promedio anual es de 4,725.2 $\mathrm{mm}$ y la temperatura media es de $24.3^{\circ} \mathrm{C}$. La vegetación predominante es una selva alta perennifolia (sensu Miranda y Hernández-X., 1963).

Los suelos son ultisoles y alfisoles, jóvenes, pardos, eutróficos, con pedones desarrollados y diferenciados, influenciados por la materia orgánica. Presentan gran abundancia de materia orgánica en los primeros centímetros de profundidad (varía entre 1.64 y $11.11 \%$ ), su capacidad de intercambio catiónico fluctúa entre 5.2 y 46.9 , y presentan toxicidad de $\mathrm{Al}$ y $\mathrm{Mn}$, con deficiencias de $\mathrm{Ca}$ y $\mathrm{Mg}$; el pH varía entre 5.3 y 6.8. Su tex t u ra es arenosa, limosa y arc illolimosa (Flores-Delgadillo et al., 1999; Sommer-Cervantes et al., 2003).

\section{Materiales y métodos}

Se recolectaron semillas de diferentes individuos de dos especies arbóreas de la selva tropical húmeda: una especie dependiente de luz (Piper auritum Kunth), y una especie tolerante a la sombra (Rollinia jimenezii Saff); para ambas especies se utilizará en adelante sólo el epíteto genérico. Las semillas fueron lavadas con hipoclorito de sodio al 5\% durante 10 minutos y enjuagadas con agua corriente; posteriormente fueron puestas a germinar en el invernadero en charolas rellenas con arena de construcción (tamaño 20/30 $\mu)$ esterilizada a vapor.

Crecimiento de plántulas en invernadero. De las plántulas que emergieron, se seleccionaron diez al azar por cada especie y se cosech a ron cuando alcanzaron una altura mínima de $10 \mathrm{~cm}$ (cosecha inicial para el experimento en invernadero). Las plantas fueron separadas en raíz, tallo y hojas; antes del secado, a las hojas se les midió el área de la lámina con un medidor de área foliar Delta-T Devices LTD. Las muestras fueron secadas durante $48 \mathrm{~h}$ en un horno a $80^{\circ} \mathrm{C}$ y se obtuvo el peso seco de cada estructura.

Las plántulas restantes se transplantaron a bolsas de $2 \mathrm{~kg}$ con el fin de aplicar los diferentes tratamientos. Para ello se consideraron dos factores (suelo y micorrización) con dos niveles cada uno. El suelo provino de selva (S) o de pastizal (P), y la micorrización consistió en inoculación con HMA $(\mathrm{M}+)$ o sin inoculación (M-). En total hubo cuatro tratamientos por especie, con 50 repeticiones por tratamiento. Para el análisis estadístico, se consideró a la especie como otro factor de variación.

Pa ra el nivel M+ se utilizó suelo sin esterilizar recolec- tado en distintos sitios de selva (SM+) o de pastizales cercanos a la Estación (PM+). El suelo se mezcló con arena estéril en una proporción de volumen 3:1 (suelo:arena). Pa ra obtener los tratamientos (SM-) y (PM-) se procedió de la misma forma que en los tratamientos anterio res; ya he cha la mezcla suelo:arena ésta se esterilizó en autocl ave sin presión durante una hora, reposando 24 horas y volviendo a ponerla en autocl ave una hora más al día siguiente.

Las plántulas se regaron las veces que fuese necesario para mantener el sustrato húmedo. Al cabo de 120 días las plántulas fueron cosechadas (14 plántulas para Rollinia en total y 17 para Piper) y separadas por estructuras; se midió su área foliar y se secaron para obtener la cosecha final.

Crecimiento de plántulas en campo. De las plántulas restantes fueron seleccionados 20 individuos por tratamiento y transplantados directamente a dos sitios en un pastizal (sin aclimatación). Para evitar alguna posible contaminación entre tratamientos por el inóculo, las plántulas fueron colocadas en bloques por tratamiento (separados al azar entre sí) en cuadros de $20 \times 20 \mathrm{~m}$ por sitio. En cada sitio y bloque se sembraron las plántulas al azar (separadas entre sí $1 \mathrm{~m}$ ) de acuerdo con el tratamiento (SM+, SM-, $\mathrm{PM}+\mathrm{y} \mathrm{PM}-; 20$ repeticiones por tratamiento).

Después de siete meses todas las plántulas fueron cosechadas (para SM+, ocho de Rollinia y siete para Piper; para SM-, cuatro de Rollinia y ocho de Piper; para PM+, siete de Rollinia y seis de Piper; y para PM-, cuatro de cada una de las dos especies). La determinación del área foliar, así como la separación de las estructuras y de los pesos secos correspondientes, se realizaron siguiendo el mismo procedimiento que en el experimento de invernadero.

Se analizó la supervivencia y el crecimiento de las plántulas en invernadero y en campo. Para esto último se llevó a cabo un análisis clásico de crecimiento (Hunt, 1982) en el que se consideraron únicamente dos cosechas, la inicial y la final, utilizando las variables (y sus fórmulas correspondientes) que se muestran en el cuadro 1.

Para evaluar el efecto de los factores sobre cada variable de respuesta se realizó un análisis de varianza (ANDEVA) de tres vías y se aplicó la prueba de comparación múltiple de medias de Tukey para discernir grupos (Zar, 1999); para ello se utilizó el paquete estadístico STATISTICA (STATSOFT INC., 2001). Antes de aplicar el ANDEVA, se verificó que los datos cumplieran con los supuestos de normalidad y homocedasticidad; de no hacerlo, fueron transformados con logaritmo natural (Zar, 1999).

La supervivencia se analizó a través de la comparación de curvas, por pares de tratamientos, de acuerdo con Pyke y Thompson (1986); los valores calculados fueron cotejados con los valores de $\chi^{2}$, con g.l. $=1$. 
Cuadro 1. Variables consideradas en los experimentos de invernadero y campo, de acuerdo con un análisis clásico de crecimiento (Hunt, 1982).

\begin{tabular}{|c|c|}
\hline Variable & Abreviatura; unidades; fórmula \\
\hline Peso seco de hojas & $\mathrm{H} ; \mathrm{g}$ \\
\hline Peso seco de tallos & $\mathrm{T} ; \mathrm{g}$ \\
\hline Peso seco total ${ }^{1}$ & PST; g; PST $=\mathrm{R}+\mathrm{T}+\mathrm{H}$ \\
\hline $\begin{array}{l}\text { Tasa relativa de } \\
\text { crecimiento }^{2}\end{array}$ & TRC; g g $^{-1}$ día $^{-1} ;$ TRC $=\left(\log \mathrm{PST}_{2}-\log \mathrm{PST}_{1}\right) /\left(\mathrm{T}_{2}-\mathrm{T}_{1}\right)$ \\
\hline $\begin{array}{l}\text { Proporción de área } \\
\text { foliar }^{3}\end{array}$ & $\mathrm{PAF} ; \mathrm{cm}^{2} \mathrm{~g}^{-1} ; \mathrm{PAF}=\mathrm{AF} / \mathrm{PST}$ \\
\hline Área foliar específica & $\mathrm{AFE} ; \mathrm{cm}^{2} \mathrm{~g}^{-1} ; \mathrm{AFE}=\mathrm{AF} / \mathrm{H}$ \\
\hline $\begin{array}{l}\text { Tasa de asimilación } \\
\text { neta }^{4}\end{array}$ & $\begin{array}{l}\text { TAN; } g \mathrm{~cm}^{-2} \text { día }^{-1} ; \\
\text { TAN }=\left(\frac{\mathrm{PST}_{2}-\mathrm{PST}_{1}}{\mathrm{~T}_{2-\mathrm{T}_{1}}}\right)\left(\frac{\log \mathrm{AF}_{2}-\log A F_{1}}{\mathrm{AF}_{2}-\mathrm{AF}_{1}}\right)\end{array}$ \\
\hline \multicolumn{2}{|c|}{$\begin{array}{l}{ }^{1} \text { Para el cálculo de esta variable, } \mathrm{R}=\text { peso seco de raíces (este dato } \\
\text { no se tomó en cuenta para el caso de campo). } \\
{ }^{2} \mathrm{~T} 1=\text { día inicial, } \mathrm{T}_{2}=\text { día final. } \\
{ }^{3} \mathrm{AF}=\text { área foliar; } \mathrm{cm}^{2} \text {. } \\
{ }^{4} \mathrm{PST}_{2} \text { y } \mathrm{PST}_{1} \text { corresponden al peso seco final e inicial, respectiva- } \\
\text { mente; } \mathrm{T}_{1}=\text { día inicial, } \mathrm{T}_{2}=\text { día final; } \mathrm{AF}_{2} \text { y } \mathrm{AF}_{1} \text { corresponden al } \\
\text { área foliar final e inicial, respectivamente. }\end{array}$} \\
\hline
\end{tabular}

\section{Resultados}

Crecimiento de plántulas en invernadero. Considerando el factor especie, solamente produjo diferencias significativas en T y PAF (cuadro 2); los valores promedio más altos de $\mathrm{T}$ correspondieron a Rollinia y los de PAF a Piper. Por su parte, el factor suelo generó diferencias significativas únicamente en $\mathrm{T}$; el valor promedio más alto correspondió a selva. Finalmente, el factor micorrización generó diferencias significativas en $\mathrm{H}$, PAF y TAN; los valores promedio más altos correspondieron a $\mathrm{M}+$ en los dos primeros casos y a M- en el último (cuadro 2).

Con respecto a las interacciones, la interacción especie $\times$ suelo fue significativa para H, PST, TRC y AFE; los valores promedio más altos se obtuvieron para Rollinia con suelo de selva (figura 1). Por otro lado, para la interacción especie $\times$ micorrización, la única que no fue significativa fue PAF; en general, las combinaciones Rollinia con micomizas y Piper sin micorrizas fueron los niveles con los valores más altos (figura 1). Con respecto a la interacción suelo $\times$ micorrización, ésta fue significativa para T, PST, TRC, PAF y TAN; los valores más altos fueron para suelo de selva con micorrizas (figura 1). Finalmente, la interacción especie $\times$ micorrización $\times$ suelo fue significativa para H, T, PAF, AFE y TAN (cuadro 2); en general el valor más alto se obtuvo para Rollinia con micorrizas en suelo de selva.

Crecimiento de plántulas en el campo. Considerando todos los tratamientos, sobresale el hecho de que el factor especie tuvo efectos significativos sobre seis de las siete variables de respuesta, siendo T la excepción; los valores promedio más altos fueron los de Piper para H, PST, TRC, AFE y

Cuadro 2. Promedios (+ E.E.) y resumen de los análisis de varianza realizados para las variables de crecimiento del experimento en invernadero. M+: con micorrizas; M-: sin micorrizas; S: suelo de selva; P: suelo de pastizal; Sp: especie; Ro: Rollina; Pi: Piper; H: peso seco hojas; T: peso seco tallo; PST: peso seco total; TRC: tasa relativa de crecimiento; PAF: proporción de área foliar; AFE: área foliar específica; TAN: tasa de asimilación neta. $P$ : nivel de significancia, ns: no significativo $(P>0.05)$. Letras diferentes indican diferencias significativas.

\begin{tabular}{|c|c|c|c|c|c|c|c|c|c|c|}
\hline Especie & $\begin{array}{l}\text { Micorri- } \\
\text { zación }\end{array}$ & Suelo & $\mathrm{n}$ & $\mathrm{H}$ & $\mathrm{T}$ & PST & TRC & PAF & AFE & TAN \\
\hline \multirow{4}{*}{$\begin{array}{l}\text { Rollinia } \\
\text { jimenezii }\end{array}$} & M+ & $S$ & 4 & $1.581 \pm 0.337 b$ & $1.459 \pm 0.252 \mathrm{C}$ & $4.959 \pm 1.060$ & $1.737 \pm 0.178$ & $10.337 \pm 1.027 b$ & $32.064 \pm 2.294 \mathrm{ab}$ & $0.0010 \pm 0.00008 \mathrm{C}$ \\
\hline & M+ & $\mathrm{P}$ & 3 & $0.673 \pm 0.061 b$ & $0.782 \pm 0.119 b c$ & $2.482 \pm 0.167$ & $1.245 \pm 0.047$ & $7.573 \pm 0.799 a b$ & $27.816 \pm 1.641 \mathrm{ab}$ & $0.0010 \pm 0.00030 \mathrm{C}$ \\
\hline & $M-$ & $S$ & 3 & $0.073 \pm 0.016 \mathrm{a}$ & $0.291 \pm 0.029 b$ & $0.690 \pm 0.022$ & $0.523 \pm 0.013$ & $3.028 \pm 0.709 a b$ & $28.553 \pm 2.398 a b$ & $0.0006 \pm 0.00010 b c$ \\
\hline & $M-$ & $P$ & 4 & $0.044 \pm 0.012 a$ & $0.172 \pm 0.023 \quad b$ & $0.496 \pm 0.075$ & $0.398 \pm 0.049$ & $6.939 \pm 1.822 \mathrm{a}$ & $128.713 \pm 59.117 \mathrm{C}$ & $0.0003 \pm 0.00004 a b$ \\
\hline \multirow{4}{*}{$\begin{array}{l}\text { Piper } \\
\text { auritum }\end{array}$} & $M_{+}$ & $S$ & 4 & $0.078 \pm 0.041 \mathrm{a}$ & $0.175 \pm 0.040 b$ & $0.706 \pm 0.114$ & $0.560 \pm 0.065$ & $9.849 \pm 6.024 a b$ & $77.821 \pm 5.588 b c$ & $0.0009 \pm 0.00020 b c$ \\
\hline & M+ & $\mathrm{P}$ & 4 & $0.405 \pm 0.107 b$ & $0.046 \pm 0.029 a$ & $0.492 \pm 0.096$ & $0.437 \pm 0.061$ & $56.115 \pm 5.429 \mathrm{c}$ & $70.375 \pm 1.658 b c$ & $0.0002 \pm 0.00003 a$ \\
\hline & $M-$ & $S$ & 5 & $0.644 \pm 0.147 b$ & $0.406 \pm 0.136 b$ & $2.462 \pm 0.605$ & $1.212 \pm 0.172$ & $10.300 \pm 2.001 b$ & $35.909 \pm 2.036 a b c$ & $0.0010 \pm 0.00030 \mathrm{C}$ \\
\hline & $M-$ & $P$ & 4 & $0.688 \pm 0.165 b$ & $0.679 \pm 0.179 b c$ & $8.770 \pm 3.454$ & $2.079 \pm 0.406$ & $2.989 \pm 1.018 \mathrm{ab}$ & $26.770 \pm 1.605 a$ & $0.0040 \pm 0.00100 \mathrm{~d}$ \\
\hline \multicolumn{2}{|l|}{ Factor } & gl & & & & & & & & \\
\hline \multicolumn{2}{|l|}{ Especie } & 1 & & $\mathrm{~ns}$ & $P<0.001 \quad(\mathrm{Ro})$ & $\mathrm{ns}$ & ns & $P<0.01 \quad(\mathrm{Pi})$ & ns & ns \\
\hline \multicolumn{2}{|l|}{ Suelo } & 1 & & ns & $P<0.05 \quad(S)$ & ns & ns & ns & ns & ns \\
\hline \multicolumn{2}{|c|}{ Micorrización } & 1 & & $P<0.01 \quad(M+)$ & $\mathrm{ns}$ & ns & ns & $P<0.001 \quad(M+)$ & ns & $P<0.05(\mathrm{M}-)$ \\
\hline \multicolumn{2}{|l|}{$S p \times S$} & 1 & & $P<0.01$ & ns & $P<0.05$ & $P<0.05$ & ns & $P<0.05$ & ns \\
\hline \multicolumn{2}{|l|}{$S p \times M$} & 1 & & $P<0.001$ & $P<0.001$ & $P<0.001$ & $P<0.001$ & ns & $P<0.001$ & $P<0.001$ \\
\hline \multicolumn{2}{|l|}{$S \times M$} & 1 & & $\mathrm{~ns}$ & $P<0.05$ & $P<0.05$ & $P<0.05$ & $P<0.001$ & ns & $P<0.01$ \\
\hline \multicolumn{2}{|l|}{$S p \times S \times M$} & 1 & & $P<0.05$ & $P<0.05$ & ns & ns & $P<0.001$ & $P<0.05$ & $P<0.001$ \\
\hline \multicolumn{2}{|l|}{ Error } & 23 & & & & & & & & \\
\hline
\end{tabular}




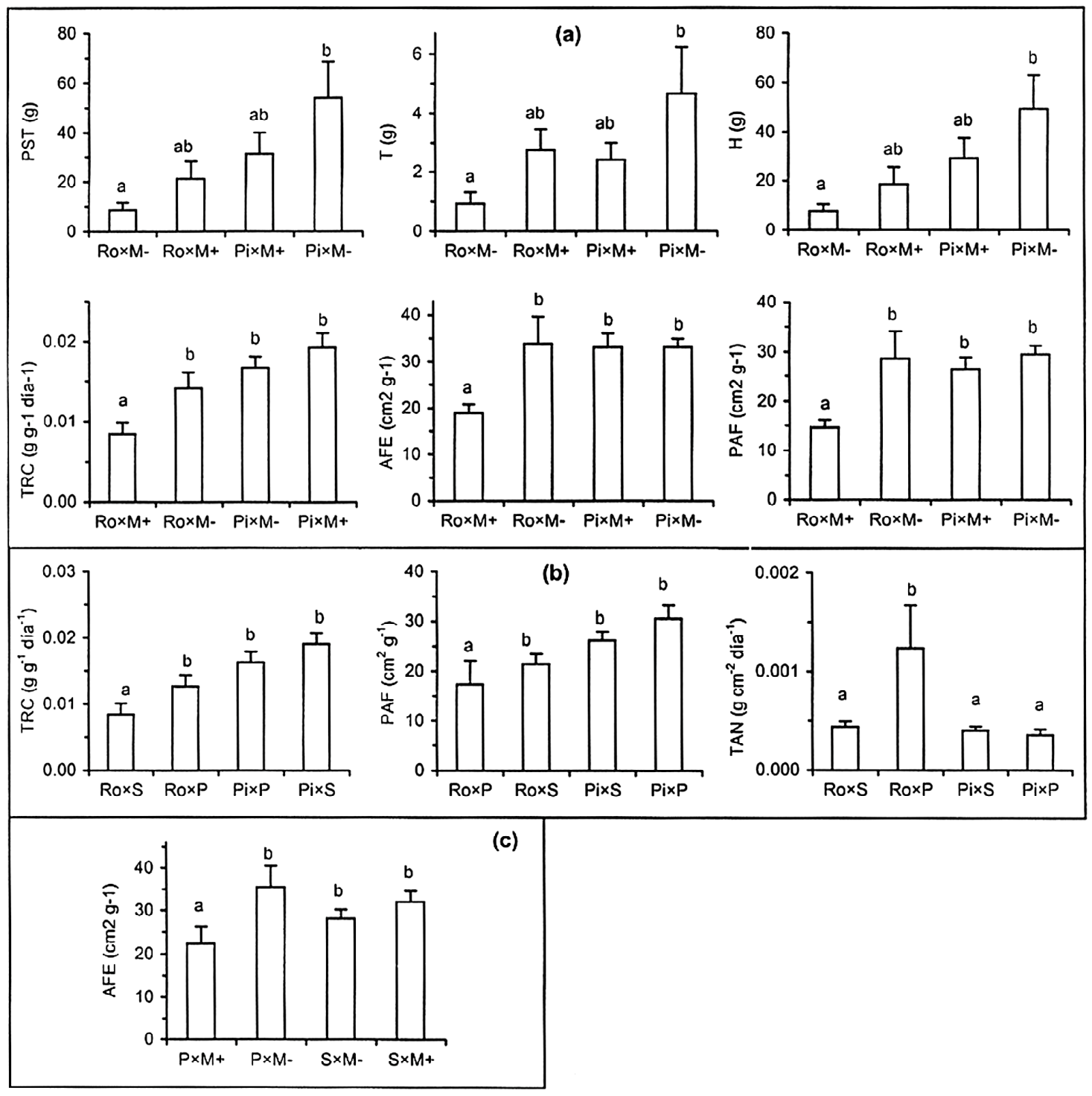

Figura 1. Promedios $( \pm 1 \mathrm{EE})$ de las variables de crecimiento que resultaron significativas en el experimento de invernadero para las dobles interacciones. Los conjuntos de gráficas corresponden a las siguientes interacciones: especie $\times$ micorrización $($ a); especie $\times$ suelo (b); suelo $\times$ micorrización (c). H: peso de hojas; T: peso de tallo; PST: peso seco total; TRC: tasa relativa de crecimiento; AFE: área foliar específica; TAN: tasa de asimilación neta. M+: tratamiento con inóculo de hongos micorrizógenos arbusculares (HMA); M-: tratamiento sin HMA. Letras diferentes indican diferencias significativas de acuerdo con la prueba de Tukey.

LAR, y el de Rollinia para TAN (cuadro 3). El factor suelo no generó diferencias significativas, mientras que el factor micorrización solamente produjo diferencias significativas para PAF, AFE y TAN; en este caso los valores más altos correspondieron al tratamiento sin micorrizas para las dos primeras, y con micorrizas para la última (cuadro 3).

La interacción especie $\times$ suelo solamente fue significativa para TRC, PAF y TAN; para TRC y TAN, Rollinia en suelo de selva tuvo los valores promedio más bajos; para PAF Rollinia en suelo de pastizal se separó de los otros niveles con los valores más bajos (figura 2). La interacción especie $\times$ micorrización fue significativa para $\mathrm{H}$, T, PST,
TRC, PAF y AFE; los valores más altos fueron en general en Piper sin micorrizas (figura 2). Por otra parte, la interacción suelo $\times$ micorrización fue significativa para AFE; esta variable tuvo los menores valores en pastizal con micorrizas (figura 2), y la triple interacción fue solamente significativa para TRC, siendo Rollinia con micorrizas en suelo de selva la que obtuvo la media menor (cuadro 3 ).

Supervivencia de plántulas. En el caso de Piper, en los tratamientos con suelo de selva, la supervivencia fue significativamente mayor $\sin$ HMA $\left(\chi^{2}=54.69, P<0.05\right)$ (figura $3 a)$; para los tratamientos con suelo proveniente del pasti- 

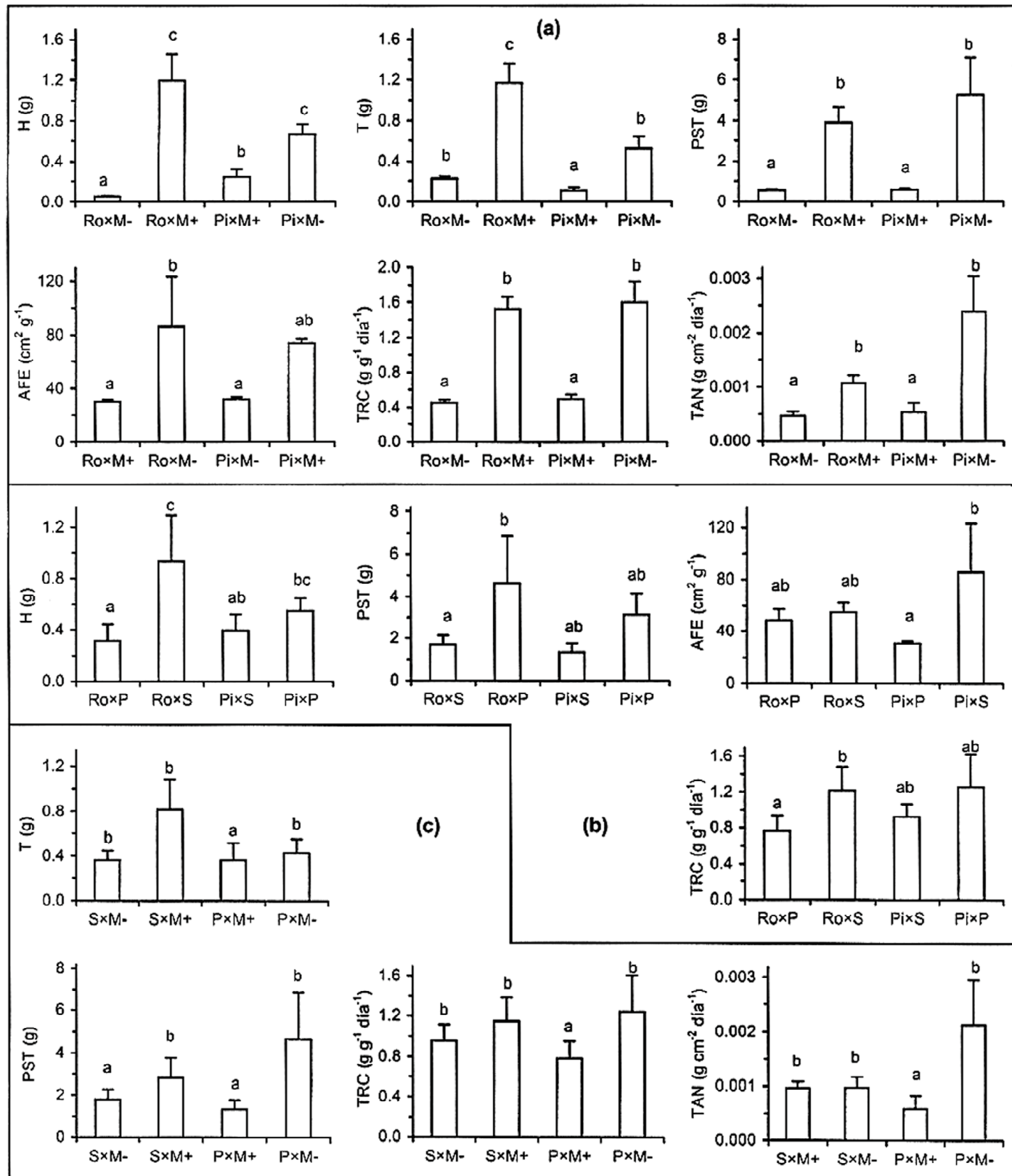

Figura 2. Promedios $( \pm 1 \mathrm{EE})$ de las variables de crecimiento que resultaron significativas para el experimento de campo para las dobles interacciones. Los conjuntos de gráficas corresponden a las siguientes interacciones: especie $\times$ micorrización (a); especie $\times$ suelo (b); suelo $\times$ micorrización $(\mathrm{c})$. H: peso de hojas; T: peso de tallo; PST: peso seco total; TRC: tasa relativa de crecimiento; PAF: proporción de área foliar; AFE: área foliar específica; TAN: tasa de asimilación neta. M+: tratamiento con inóculo de hongos micorrizógenos arbusculares (HMA); M-: tratamiento sin HMA. Letras diferentes indican diferencias significativas de acuerdo con la prueba de Tukey.

zal, la supervivencia fue significativamente mayor con HMA $\left(\chi^{2}=12.47, P<0.05\right)$ (figura 3b). Para Rollinia, la supervivencia fue significativamente mayor en los tratamientos micorrizados, sin importar el origen del suelo $\left(\chi^{2}=\right.$ $13.08, P<0.05$ para suelo de pastizal; $\chi^{2}=37.25, P<0.05$ con suelo de selva) (figuras 3 c y $3 d$ ).

\section{Discusión}

A diferencia de lo que ocurrió en el experimento de invernadero, en el campo el factor especie produjo diferencias significativas; en el primer caso Rollinia tuvo los valores promedio más altos, mientras que Piper los tuvo en el campo. Respecto al factor suelo, éste no fue significativo; 

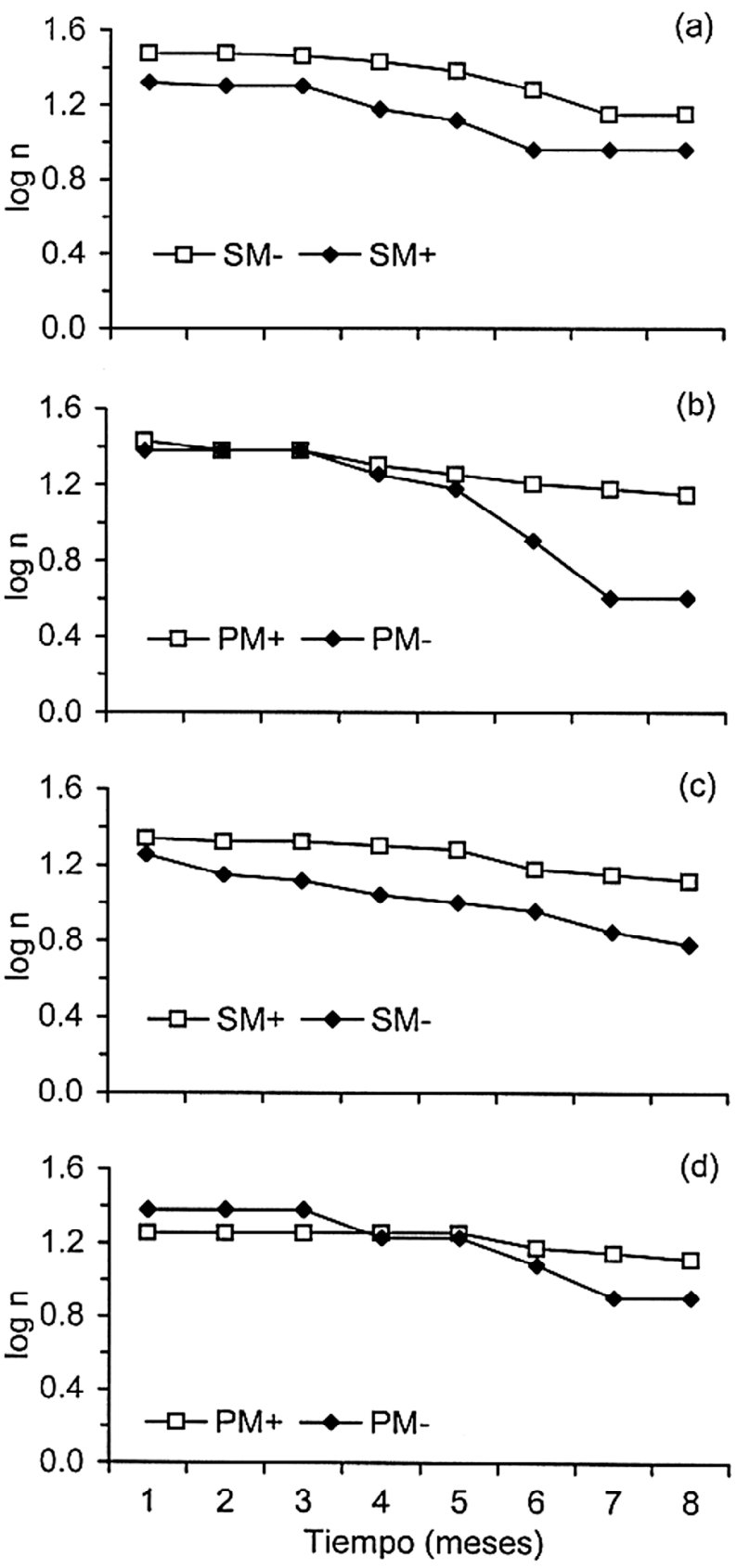

Figu ra3. Supervivencia en campo de las plántulas de Piper auri tum (a, b) y Rollinia jimenezii (c, d) a lo largo del tiempo. S: suelo de selva; P: suelo de pastizal; M+: tratamiento con inóculo de hongos micorrizógenos arbusculares (HMA); M-: tratamiento sin HMA.

sin embargo, una vez trasplantadas al campo, las plántulas de Rollinia sobrevivieron más sin importar el origen del suelo, mientras que las de Piper tuvieron mayor supervivencia con suelo de pastizal.

Se ha determinado que las especies de rápido crecimiento responden positiva y rápidamente a los cambios en el ambiente (Grime et al., 1986; Huante et al., 1992); éste parece ser el caso de Piper. Por otra parte, se ha reportado que las especies con un crecimiento más lento (como Rollinia) tienden a resistir los cambios en las condiciones ambientales más que responder a ellos, positiva o negativamente. Sin embargo, en nuestro experimento no fue así, ya que en el campo esta especie tuvo valores más bajos que los obtenidos en invernadero.

Con respecto al factor micorrización, éste tuvo efectos significativos sobre las plántulas, particularmente en variables relacionadas con el peso de la hoja (H y PAF), su actividad fotosintética (indirectamente indicada por TAN) y su grosor (indirectamente indicado por AFE) (Rodríguez, 2006); en invemadero, los mayo res valorespromedio fueron obtenidos en $\mathrm{H}$ y PAF en los tratamientos con mico-rrizas, mientras que en campo PAF y AFE obtuvieron los mayores valores en los tratamientos sin el inóculo micorrícico. En el caso de TAN, los valores más altos correspondieron a los tratamientos sin micorrizas en invernadero y con micorrizas en campo. Ello indica que en el invernadero, las plantas son poco eficientes, aun con micorrizas, pues tuvieron bajo peso con respecto al área foliar. Por otro lado, en el campo podría considerarse que hay indicios indirectos de que hubo una mayor actividad fotosintética con la presencia de las micorrizas, en virtud de los valores mayo res de TAN.

Considerando la interacción especie $\times$ micorrización, la respuesta de Rollinia en la TRC fue favorable en el invernadero, pero no en campo; en cambio, en Piper sí hubo un efecto positivo por la micorrización en la TRC en campo. Esto indica que la presencia de los HMA en Rollinia representó un gasto energético que disminuyó su TRC.

En el caso de Piper, las hojas fueron más delgadas en campo (mayor AFE, Huante et al., 1992), lo que les permitió una más eficiente captación de radiación (Morrison y Reeke, 1995; Rodríguez, 2006), y repercutió en una mayor fijación de biomasa, pues los valores de PAF fueron menores siempre y cuando tuvieran micorrizas. En Rollinia, si bien hubo cambios, los valores tendieron a ser menores que en invernadero, lo cual indica que se trata de una especie menos eficiente en comparación con Piper en presencia de micorrizas.

La interacción micorrización $\times$ especie fue diferente significativamente en la mayoría de las variables, tanto en campo como en invernadero. Esto puede deberse a la capacidad de respuesta diferencial (mycorrhizal dependen cy o mycorrhizal responsiveness) de una especie de planta a una especie de hongo, lo que ha sido señalado como un factor importante en el éxito de la relación mutualista (Habte y Manjunath, 1991).

En otras investigaciones se ha encontrado un incremento en la biomasa de los hospederos debido a la inoculación por HMA (Hart y Reader, 2004; Lekberg y Koide, 2005). En nuestro estudio esto ocurrió en varias ocasiones para el 
Cuadro 3. Promedios (+ E.E.) y resumen de los análisis de varianza realizados para las variables de crecimiento del experimento en el campo. M+: con micorrizas; M-: sin micorrizas; S: suelo de selva; P: suelo de pastizal; Sp: especie; Ro: Rollina; Pi: Piper; H: peso seco hojas; T: peso seco tallo; PST: peso seco total; TRC: tasa relativa de crecimiento; PAF: proporción de área foliar; AFE: área foliar específica; TAN: tasa de asimilación neta. $P$ : nivel de significancia, ns: no significativo $(P>0.05)$. Letras diferentes indican diferencias significativas.

\begin{tabular}{|c|c|c|c|c|c|c|c|c|c|c|}
\hline Especie & $\begin{array}{l}\text { Micorri } \\
\text { zación }\end{array}$ & Suelo & & $\mathrm{H}$ & $\mathrm{T}$ & PST & TRC & PAF & AFE & TAN \\
\hline Rollinia & $M_{+}$ & S & 8 & $11.88 \pm 3.98$ & $2.40 \pm 0.67$ & $14.28 \pm 4.15$ & $0.005 \pm 0.001 \mathrm{a}$ & $18.64 \pm 0.96$ & $24.34 \pm 1.732$ & $0.0005 \pm 0.00007$ \\
\hline \multirow[t]{3}{*}{ jimenezii } & i $\quad M+$ & $P$ & 7 & $26.00 \pm 14.39$ & $3.14 \pm 1.38$ & $29.14 \pm 14.14$ & $0.012 \pm 0.002 b$ & $9.98 \pm 1.96$ & $12.64 \pm 1.677$ & $0.0020 \pm 0.00060$ \\
\hline & $M-$ & $S$ & 4 & $8.75 \pm 3.33$ & $1.40 \pm 0.74$ & $10.15 \pm 3.95$ & $0.014 \pm 0.002 b$ & $26.94 \pm 5.73$ & $31.61 \pm 6.499$ & $0.0004 \pm 0.00006$ \\
\hline & $M-$ & $\mathrm{P}$ & 4 & $6.75 \pm 4.80$ & $0.45 \pm 0.12$ & $7.20 \pm 4.75$ & $0.014 \pm 0.003 b$ & $30.29 \pm 10.28$ & $36.20 \pm 10.617$ & $0.0004 \pm 0.00020$ \\
\hline \multirow{4}{*}{$\begin{array}{l}\text { Piper } \\
\text { auritum }\end{array}$} & $M_{+}$ & S & 7 & $37.29 \pm 14.41$ & $3.04 \pm 1.02$ & $40.33 \pm 14.51$ & $0.021 \pm 0.002 b$ & $23.99 \pm 2.63$ & $32.54 \pm 3.400$ & $0.0004 \pm 0.00009$ \\
\hline & $M+$ & $P$ & 6 & $19.17 \pm 7.28$ & $1.67 \pm 0.37$ & $20.83 \pm 7.61$ & $0.017 \pm 0.002 b$ & $29.27 \pm 4.38$ & $33.77 \pm 5.460$ & $0.0004 \pm 0.00009$ \\
\hline & $M-$ & S & 8 & $58.00 \pm 20.23$ & $6.06 \pm 2.23$ & $64.06 \pm 21.43$ & $0.017 \pm 0.002 b$ & $28.00 \pm 2.32$ & $32.25 \pm 2.600$ & $0.0004 \pm 0.00003$ \\
\hline & M- & $\mathrm{P}$ & 4 & $32.00 \pm 6.07$ & $1.83 \pm 0.67$ & $33.83 \pm 5.56$ & $0.016 \pm 0.002 b$ & $32.46 \pm 2.13$ & $34.83 \pm 1.890$ & $0.0003 \pm 0.00002$ \\
\hline \multicolumn{4}{|l|}{ Factor } & & & & & & & \\
\hline \multicolumn{2}{|l|}{ Especie } & 1 & & $P<0.01(\mathrm{Pi})$ & ns & $P<0.01(\mathrm{Pi})$ & $P<0.001(\mathrm{Pi})$ & $P<0.01(\mathrm{Pi})$ & $P<0.01(\mathrm{Pi})$ & $P<0.05($ Ro $)$ \\
\hline \multicolumn{2}{|l|}{ Suelo } & 1 & & $\mathrm{~ns}$ & ns & ns & ns & ns & ns & ns \\
\hline \multicolumn{2}{|c|}{ Micorrización } & 1 & & ns & ns & ns & ns & $P<0.01(\mathrm{M}-)$ & $P<0.01(M-)$ & $P<0.01(\mathrm{M}+)$ \\
\hline \multicolumn{2}{|c|}{$\mathrm{Sp} \times \mathrm{S}$} & 1 & & ns & ns & ns & $P<0.05$ & $P<0.05$ & ns & $P<0.05$ \\
\hline \multicolumn{2}{|l|}{$S p \times M$} & 1 & & $P<0.05$ & $P<0.05$ & $P<0.05$ & $P<0.05$ & $P<0.05$ & $P<0.01$ & $\mathrm{~ns}$ \\
\hline \multicolumn{2}{|l|}{$S \times M$} & 1 & & $\mathrm{~ns}$ & ns & ns & ns & ns & $P<0.05$ & ns \\
\hline \multicolumn{2}{|c|}{$S p \times S \times M$} & 1 & & ns & ns & ns & $P<0.05$ & ns & ns & ns \\
\hline \multicolumn{2}{|l|}{ Error } & 40 & & & & & & & & \\
\hline
\end{tabular}

experimento en invernadero, ya que se observa que en las fuentes de variación que involucran el factor micorrización fueron significativas para H, T, PST y TRC (cuadro 2). En el campo, este efecto en la biomasa de las plántulas fue determinado por la especie, ya que hubo diferencias significativas en H, PST y TRC, y ello es reforzado por el resultado de la interacción suelo $\times$ especie $\times$ micorrización para la TRC. Esto ocurrió claramente en el caso de Piper.

Antes de realizar un trabajo de restauraión a gran escala, se ha suge rido la necesidad de desarrollar pruebas experimentales con especies nativas para estudiar su adaptabilidad, a partir de su crecimiento y supervivencia (Haggar et al., 1998), así como su respuesta fisiológica ante dife rentes condiciones y recursos (Medina, 1995) debido a que las especies tropicales han mostrado poseer un amplio intervalo de respuesta ante la va riación de las condiciones ambientales (Haggar et al., 1998). Este estudio es un avance en ese sentido, al probar la potencialidad de las plántulas de dos especies para ser utilizadas en la restauración ecológic a.

Estudios realizados en sistemas tropicales demuestran la necesidad de usar a los HMA como una herramienta en la restauración de sitios perturbados. Pouyú-Rojas y Siqueira (2000) encontraron que plántulas inoculadas con HMA tuvieron valores mayores en altura y biomasa que plantas no inoculadas, mientras que Allen et al. (2003), en un estudio realizado en una selva seca estacional de la península de Yucatán, encontraron que plantas nat ivas inoculadas con HMA y transplantadas a un sitio perturbado tuvieron valores mayores en altura, cobertura, biomasa, diámetro del tallo y en su tasa de crecimiento, con respecto a los obtenidos con plantas sin inóculo. De acuerdo con los resultados de este trabajo, la inoculación previa de las plántulas de especies pioneras (dependientes de luz) y sucesionalmente tardías (tolerantes a la sombra) con hongos micorrizógenos arbusculares es recomendable en la restauración ecológica, ya que las plántulas tendrían una ventaja con respecto a las no micorrizadas; los resultados para TAN, TRC para Piper en el campo y la mayor supervivencia de Rollinia también en el campo apoyan esta conclusión. En el caso de Rollinia, la inoculación debería realizarse previamente al trasplante, porque si bien las diferencias encontradas no fueron significativas, en general sí fueron más altos los valores en algunas variables de respuesta.

Las primeras especies de plantas nativas que se deben introducir son las que posean altas tasas de crecimiento como las pioneras, ya que éstas pueden facilitar el proceso sucesional y promover el establecimiento de otras especies, hasta alcanzar una estructura y una composición semejantes a la vegetación original (Guariguata y Ostertag, 2001; Leopold et al., 2001). La respuesta favorable de Piper al trasplante hace de ella una especie útil en términos de la restauración, independientemente de que no haya respondido a la micorrización. Si bien dicha introducción 
de especies dependientes de luz propicia el inicio de la sucesión secundaria, es importante también considerar la persistencia o la ausencia de la cobertura de los pastos, así como el número de plántulas colonizadoras que lleguen al sistema a través de semillas originarias de otras fuentes externas (Maluf de Souza y Ferreira, 2004). Esto generará las condiciones necesarias para el establecimiento de otras especies, principalmente nativas y leñosas de la región (Engel y Parrota, 2001; Guariguata y Ostertag; 2001; Meli, 2003), como podría ser el caso de Rollinia, la cual mostró un crecimiento más lento después del trasplante, pero una mayor supervivencia en los tratamientos con HMA.

\section{Agradecimientos}

Los autores agradecen a Oswaldo Núñez, Alejandro Astudillo, Juan Carlos Peña, B raulio Gómez Chagala, Zenet García y al Sr. Juan Gómez por su ayuda en el trabajo de campo. Asimismo, queremos agradecer a Consuelo Bonfil por sus valiosos comentarios al primer manuscrito, así como a los revisores anónimos por sus suge rencias que enriquecieron en mu cho este trabajo. Esta investigación fue realizada gracias al apoyo de los proyectos PAPIIT-UNAM 205599 y 235402 y SEMARNATCONACYT 2002 C01-668.

\section{Literatura citada}

Allen E.B., Allen M.F., Egerton-Warburton L., Corkidi L. y Gómez-Pompa A. 2003. Impacts of early- and late-seral mycorrhizae during restortion in seasonal tropical forest, Mexico. Ecological Applications 13:1701-1717.

Aronson J.C. y Le Floc'h E. 1996. Vital landscape attributes: missing tools for restoration ecology. Restoration Ecology 4:377-387.

Aziz T., Sylvia D.M. y Doren R.F. 1995. Activity and species composition of arbuscular mycorrhizal fungi following soil removal. Ecological Applications 5:776-784

Bradshaw A.D. 1997. The importance of soil ecology in restoration science. En: Urbanska K.M., Webb N.R. y Edwards P.J. Eds. Restoration Ecology and Sustainable Development, pp. 33-64, Cambridge University Press, Cambridge.

Cuenca G., De Andrade Z. y Escalante G. 1998a. Diversity of Glomalean spores from natural, disturbed and revegetated communities growing on nutrient-poor tropical soils. Soil Biology and Biochemistry 30:711-719.

Cuenca G., De Andrade Z. y Escalante G. 1998b. Arbuscular mycorrhizae in the rehabilitation of fragile degraded tropical lands. Biology and Fertility of Soils 26:107-111.

Cuenca G., De Andrade Z., Lovera M., Fajardo L. y Meneses E. 2004. The effect of two arbuscular mycorrhizal inocula of contrasting richness and the same mycorrhizal potential on the growth and survival of wild plant species from La Gran Sabana, Venezuela. Canadian Journal of Botany 82:582-589.

Dirzo R. 2001. Tropical forests. En: Chapin III F.S., Sala O.E. y Huber-Sannwald E. Eds. Global Biodiversity in a Changing Environment: Scenarios for the 21st Century, pp. 251-276,
Springer-Verlag, Berlín.

Dobson A.P., Bradshaw A.D. y Baker J.M. 1997. Hopes for the future: restoration ecology and conservation biology. Science 277:515-522.

Engel V.L. y Parrota J.A. 2001. An evaluation of direct seeding for reforestation of degraded lands in central São Paulo state, Brazil. Forest Ecology and Management 152:169-181.

Flores-Delgadillo L., Sommer-Cervantes I., Alcalá-Martínez J.R. y Álvarez-Sánchez J. 1999. Estudio morfogenético de algunos suelos de la región de Los Tuxtlas, Veracruz, México. Revista Mexicana de Ciencias Geológicas 16:81-88.

García M.C. 1988. Lanscape ecological approach for forest conservation. A case in Los Tuxtlas, Veracruz, Mexico. Tesis de Maestría, International Institute for Aerospace Survey and Earth Sciences, Enschede, Holanda, 163 pp.

Geist H.J. y Lambin E.F. 2001. What drives tropical deforestation? A meta-analysis of proximate and underlying causes of deforestation based on sub-national case study evidence. LUCC International Project Office, Louvain-la-Neuve, Bélgica.

Geist H.J. y Lambin E.F. 2002. Proximate causes and underlying driving forces a nonrenewable resource. Science 177:762-765.

González-Soriano E., Dirzo R. y Vogt R.C. 1997. Historia Natural de Los Tuxtlas. Universidad Nacional Autónoma de México, Comisión Nacional para el Conocimiento y Uso de la Biodiversidad, México, D.F.

Grime J.P., Crack J.E. y Rincón E. 1986. The ecological significance of plasticity. En: Jennings D.H. y Trewavas A.J. Eds. Plasticity in Plants, pp 5-29, Symposia of the Society for Experimental Biology No. 40. The Company of Biologists, Cambridge.

Guariguata M.R. y Ostertag R. 2001. Neotropical secondary forest succession: changes in structural and functional characteristics. Forest Ecology and Management 148:185-206.

Guevara S., Laborde J. y Sánchez-Ríos G. 2004. Los Tuxtlas, el Paisaje de la Sierra. Instituto de Ecología, A.C., Xalapa.

Habte M. y Manjunath A. 1991. Categories of vesicular-arbuscular mycorrhizal dependency of host species. Mycorrhiza 1:312.

Haggar J.P., Briscoe C.B. y Butterfield R.P. 1998. Native species: a resource for the diversification of forestry production in the lowland humid tropics. Forest Ecology and Management 106:195-203.

Hart M.M. y Reader R.J. 2004. Do arbuscular mycorrhizal fungi recover from soil disturbance differently? Tropical Ecology 45:97-111.

Hart M.M., Reader R.J. y Klironomos J.N. 2003. Plant coexistence mediated by arbuscular mycorrhizal fungi. Trends in Ecology and Evolution 18:418-423.

Haselwandter K. 1997. Soil micro-organisms, mycorrhiza, and restoration ecology. En: Urbanska K.M., Webb N.R. y Edwa rds P.J. Eds. Restoration Ecology and Sustainable Development, pp. 65-80, Cambridge University Press, Cambridge.

Hérault B., Honnay O. y Thoen D. 2005. Evaluation of the ecological restoration potential of plant communities in Norway spruce plantations using a life-trait based approach. Journal of Applied Ecology 42:536-545.

Hobbs R.J. y Norton D.A. 1996. Towards a conceptual framework for restoration ecology. Restoration Ecology 4:93-110. 
Huante P., Rincón E. y Gavito M. 1992. Root system analysis of seedlings of seven tree species from tropical dry forest in Mexico. Trees 6:77-82.

Hughes F.R., Kauffman J.B. y Jaramillo V. 2000. Ecosystem-scale impacts of deforestation and land use in a humid tropical region of Mexico. Ecological Applications 10:515-527.

Hunt R. 1982. Plant Growth Analysis. Edward Arnold, Londres.

Janos D.P. 1996. Mycorrhizas, succession, and the rehabilitation of deforested lands in the humid tropics. En: Frankland J.C., Magan N. y Gadd G.M. Eds. Fungi and Environmental Change, pp. 129-162, Cambridge University Press, Cambridge.

Lekberg Y. y Koide R.T. 2005. Is plant performance limited by abundance of arbuscular mycorrhizal fungi? A meta-analysis of studies published between 1988 and 2003. New Phytologist 168: $189-204$.

Leopold A.C., Andrus R., Finkeldey A. y Knowles D. 2001. Attempting restoration of wet tropical forest in Costa Rica. Forest Ecology and Management 142:243-249.

Lüttge U. 1997. Physiological Ecology of Tropical Plants. Springer-Verlag, Berlín.

Maluf de Souza F. y Ferreira J.L. 2004. Restoration of seasonal semideciduous forests in Brazil: influence of age and restoration design on forest structure. Forest Ecology and Management 191:185-200.

Mangan S.A., Eom A.H., Adler G.H., Yavitt J.B. y Herre E.A. 2004. Diversity of arbuscular mycorrhizal fungi across a fragmented forest in Panama: insular spore communities differ from mainland communities. Oecologia 141:687-700.

Medina E. 1995. Physiological ecology of trees and application to forest management. En: Lugo A.E. y Lowe E. Eds. Tropical Forest: Management and Ecology, pp. 289-307, Ecological Studies 112, Springer-Verlag, Berlín.

Meli P. 2003. Restauración ecológica de bosques tropicales. Veinte años de investigación académica. Interciencia 28:581589.

Miranda F. y Hernández-X. E. 1963. Los tipos de vegetación de México y su clasificación. Boletín de la Sociedad Botánica de México 29:20-179.

Morrison D. y Reekee E. 1995. Pattern of defoliation and its effect photosynthesis capacity on Oenothera blennies. Journal of Ecology 83:759-767.

Perry D.A. y Amaranthus M.P. 1990. The plant-soil bootstrap: microorganisms and reclamation of degraded ecosystems. En: Berger J.J. Ed. Environmental Restoration, pp. 94-102, Island Press, Washington D.C.

Picone C. 2000. Dive rsity and abundance of arbuscularmycorrhizal fungus spores in tropical forest and pasture. Biotropica 32:734-750.

Pouyú-Rojas E. y Siqueira J.O. 2000. Micorriza arbuscular e fertilização do solo no desenvolvimento pós-transplante de mudas de sete espécies florestais. Pesquisa Agropecuária Brasileira Brasilia 35:103-114.

Pyke D. y Thompson J. 1986. Statistical analysis of survival and removal rate experiments. Ecology 67:240-245.

Rodríguez J. 2006. Efectos de la defoliación inducida sobre el crecimiento de tres arbustos ribereños de la Sierra Tarahumara bajo condiciones de crecimiento en rizotrón. Tesis de Maestría, Universidad Nacional Autónoma de México, México, D.F., 79 pp.

SER [Society for Ecological Restoration International Science \& Policy Working Group]. 2004. The SER International Primer on Ecological Restoration.

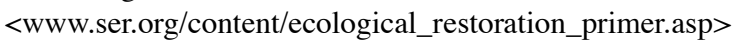

Siqueira J.O., Carneiro M.A. C., Curi N., da Silva R.S.C. y Davide A.C. 1998. Mycorrhizal colonization and mycotrophic growth of native woody species as related to successional groups in Southeastern Brazil. Forest Ecology and Management 107:241-252.

Smith S.E. y Read D.J. 1997. Mycorrhizal Symbiosis. 2a. ed. Academic Press, Cambridge.

Sommer-Cervantes I., Flores-Delgadillo L. y Gutiérrez-Ruiz M. 2003. Caracterización de los suelos de la Estación de Biología Tropical Los Tuxtlas. En: Álvarez-Sánchez J. y NaranjoGarcía E. Eds. Ecología del Suelo en la Selva Tropical Húmeda de México, pp. 17-67, Instituto de Ecología, A.C., Xalapa.

STATSOFT INC. 2001. STATISTICA for Windows. Ver. 6.0. $<$ www.statsoft.com>

Van der Heijden M.G.A., Boller T., Wiemken A. y Sanders I.R. 1998a. Different arbuscular mycorrhizal fungal species are potential determinants of plant community structure. Ecology 79:2082-2091.

Van der Heijden M.G.A., Klironomos J.N., Ursic M., Moutoglis P., Streitwolf-Engel R., Boller T., Wiemken A. y Sanders I.R. 1998b. Mycorrhizal fungal diversity determines plant biodiversity, ecosystem variability and productivity. Nature 396:69-72.

Varela L. y Guadarrama P. 2003. El papel de las micorrizas en los trópicos. En: Álvarez-Sánchez J. y Naranjo-García E. Eds. Ecología del Suelo en la Selva Tropical Húmeda de México, pp. 274-285, Instituto de Ecología, A.C., Xalapa.

Varma A. y Hock B. 1999. Mycorrhiza. Structure, Function, Molecular Biology and Biotechnology. Springer-Verlag, Berlín.

Wardle D.A. 2002. Communities and Ecosystems. Linking the Aboveground and Belowground Components. Princeton University Press, Nueva Jersey.

Young T. 2000. Restoration ecology and conservation biology. Biological Conservation 92:73-83.

Zar J. 1999. Biostatistical Analysis. Prentice-Hall, Upper Saddle River, Nueva Jersey.

Recibido: 21 de septiembre de 2005

Versión corregida: 9 de abril de 2007

Aceptado: 13 de abril de 2007 